\title{
Extensor expansion of the rheumatoid hand
}

\author{
K. M. BACKHOUSE
}

From the Royal College of Surgeons of England, the Chelsea and Kensington Rheumatology Unit, and the Royal London Homeopathic Hospital

The problem of ulnar drift in the rheumatoid hand has interested rheumatologists and hand surgeons for many years and almost as many theories as to cause have been postulated as there are structures in the vicinity of the joints concerned. To present yet another, without necessarily invalidating the rest, can be supported on the grounds that the metacarpophalangeal joints are extremely complicated in both anatomy and function and that several factors can and usually do play a part in the causation of the deformity.

Brewerton (1965) has postulated that laxity of the collateral ligaments consequent upon rheumatoid disease is an important factor, whereas Smith, Juvinall, Bender, and Pearson (1964) and Flatt (1968) favour the role of flexor tendon pull. It is necessary to accept as a basic concept that disruption of the normal supporting and controlling structures of the joint is necessary to allow the abnormal range of mobility in the deformities. This may be due to synovial bulk producing stretch of these structures, intrinsic change in them, or both. Having established abnormal ranges of mobility, structures outside the joint are then more able to play a part in their increase; for instance the radial deviation at the wrist shown by Shapiro (1968) and Pahle and Raunio (1969), and the zig-zag effect of Stack and Vaughan-Jackson (1971).

The present study is divided into two parts. An anatomical investigation was made of the digital metacarpophalangeal joints in normal subjects from functional and morphological points of view. Digital function was analysed with particular reference to the total hand functions described by Napier (1956) and Landsmeer (1962). The asymmetrical features of the MCP joints, already noted by Backhouse (1968) in his study of muscle control of the joints, were analysed in a series of fifty dissecting room subjects (100 hands). The normal findings were then related to observations on the extensor apparatus of the hands of twenty patients at operations on the MCP joints in which rheumatoid joint disorganization had occurred.

Mechanical studies on the lateral strength of the extensor expansion have been carried out on fixed and fresh post mortem material.

Accepted for publication September 3, 1971

\section{NORMAL ANATOMY}

Digital movement patterns

Power movements in the hand are characterized by the fingers being flexed at all joints, to bring them into an oblique flexion line against the thenar eminence. In order to achieve this, ulnar deviation and rotation occur at the metacarpophalangeal joints of all fingers and there is some flexion at the carpometacarpal joint $\vec{N}$ of the ring and rather more of the little fingers. The $\mathrm{O}$ ulnar deviation may be so marked in some subjects

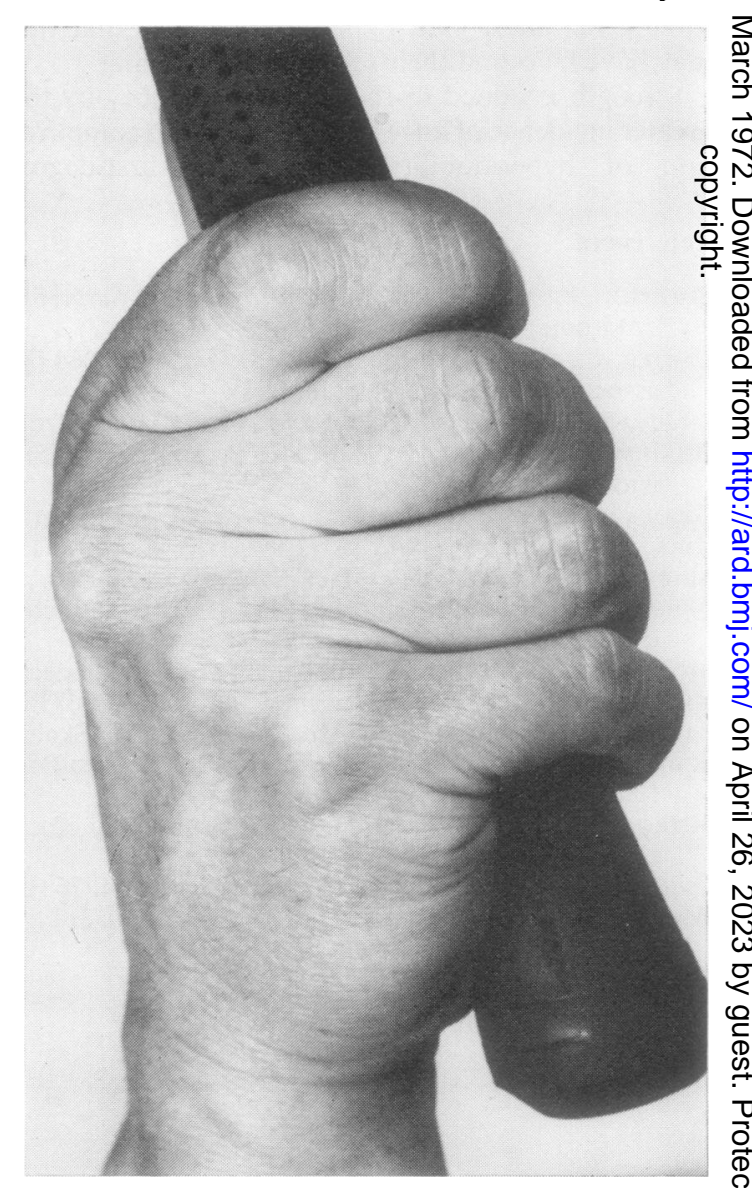

FIG. 1 Power grip in a normal hand to show the positions of the carpometacarpal and metacarpophalangeal joints. 
(particularly those with short thumbs) as to resemble the hand of a patient with rheumatoid ulnar drift (Fig. 1).

Precision movements are essentially of the metacarpophalangeal joints, the interphalangeals being held in fixed partial flexion. If the precision movement involves finger-thumb pad approximation (i.e. precision grip), then ulnar deviation and rotation (digital opposition) take place at the finger metacarpophalangeal joints as well as in the thumb (Fig. 2). Only by this means can proper pad-to-pad contact occur.

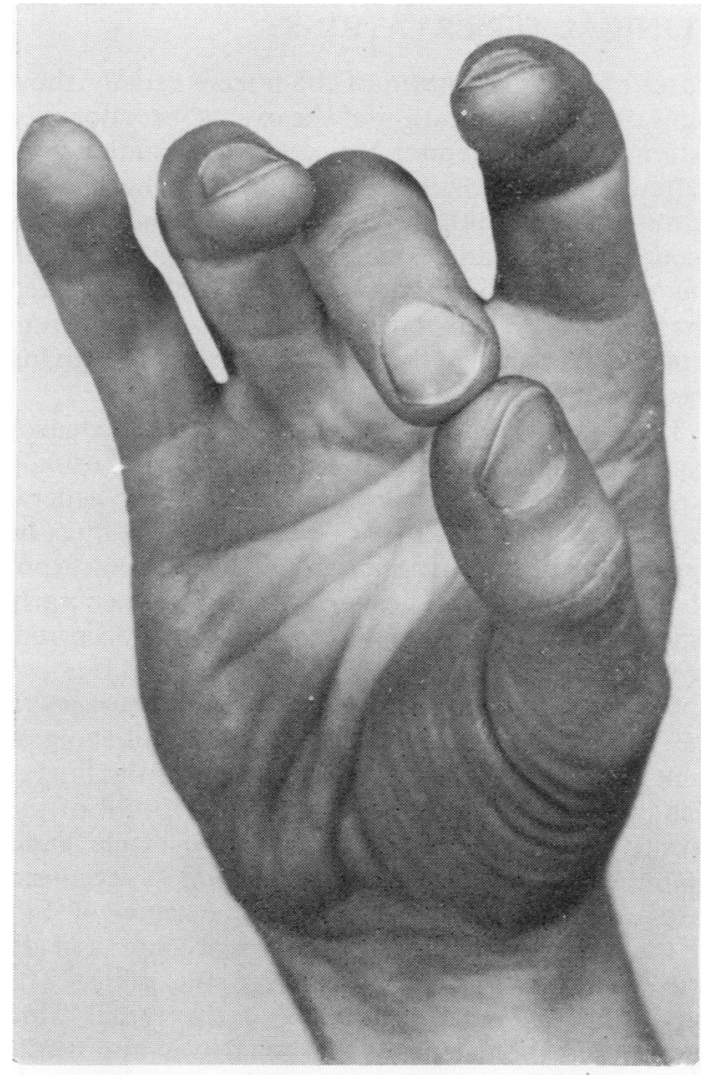

FIG. 2 Precision grip between thumb and middle finger. Note the digital, as well as thumb, opposition which bring finger and thumb pads into direct contact.

The baggage or hook grip is the only one utilizing pure digital flexion towards a single point proximal to the wrist in accord with the classical anatomical description. It is mainly limited to carrying baggage with handles; many transverse grip handles formerly in use on machinery, etc., have been replaced to allow the more comfortable and efficient oblique power grip.

Pinch grips can properly be considered to be of two different types. One is merely a firmer pad-to-pad precision grip and, although a valuable clinical test of muscle function, its use is much less than the other, which perhaps should be called only the 'key grip' to avoid confusion. This latter consists of thumb pressure onto the radial side of the index finger, which must therefore be radially deviated to a comparable degree of power. It will be apparent from a consideration of these grip patterns that practically all hand usage will require active metacarpophalangeal flexion. Ulnar deviation and rotation at the metacarpophalangeal joints are associated with flexion in all power activities, if baggage-carrying and key grips are excluded, and in many precision movements. Although it will obviously vary considerably from person to person, it is estimated that some 80 per cent. of all digital flexor movement will require ulnar deviation and rotation at the metacarpophalangeal joints.

\section{Morphology}

Over a 3-year period the extensor expansions of the hands of dissecting room subjects were examined in Charing Cross Hospital Medical School and the number of subjects was made up to fifty from other sources. The expansions were dissected off the fingers by cutting the extensor tendon proximally and removing the lumbrical and interossei from their origins (Fig. 3). Without a lead being given, student

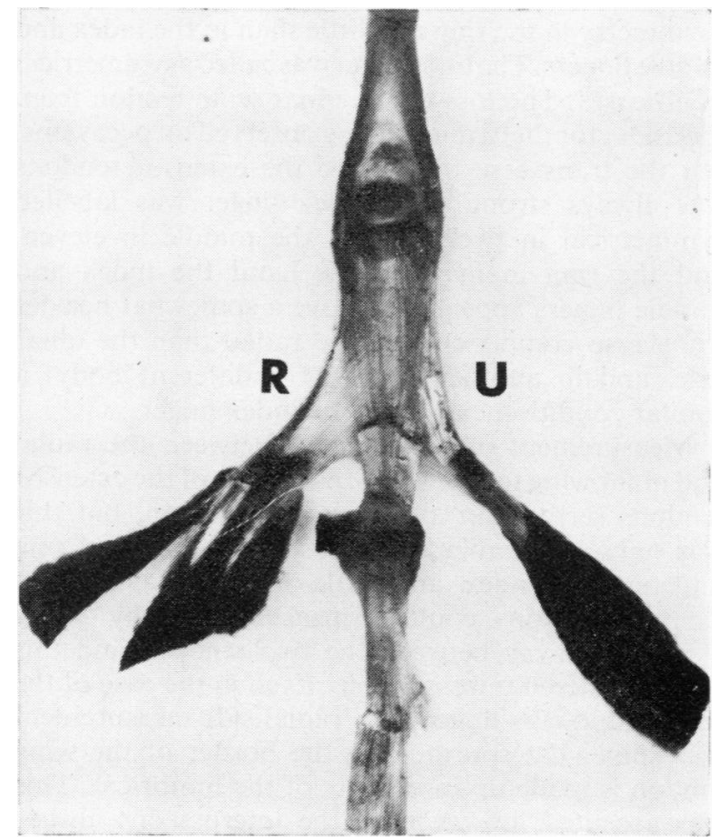

FIG. 3 Extensor expansion of a middle finger. Note the asymmetry between the two sides; the ulnar $(U)$ interosseous is densely bound to the extensor tendon, whilst the radial one (with the lumbrical) ( $R$ ) has a relatively thin triangular zone over the metacarpophalangeal joint. 
observers were invited to estimate whether the ulnar or radial-sided interosseous muscle had the denser and firmer attachment to the extensor tendon by the transverse fibres of the hood. In the majority of cases, the picture presented was in accord with that shown in the specimen in Fig. 3. The wing tendons ran alongside the extensor tendon which they gradually approached as they passed distally. The intervening part of the extensor hood, made of essentially transverse fibres, was relatively thin on the radial side. On the ulnar side the transverse fibres were much shorter and denser. The ulnar-sided interosseous was therefore bound more intimately and powerfully to the extensor tendon over the metacarpophalangeal joint than the radial interosseous and lumbrical tendons.

There was considerable variation from subject to subject and also among the fingers of a single individual. The two hands of one person were often markedly dissimilar. Observations were essentially subjective and numerical accuracy could be only approximate as fingers showed a smooth gradation from obvious asymmetry to those in which it was not possible to make such a judgement. If there was any doubt they were put down as symmetrical.

Of the 100 hands, thirteen were put down as having symmetrical expansions on one or more fingers. The remaining 87 showed an asymmetrical picture broadly similar to that shown in Fig. 3 in all fingers. As an overall observation there was a trend to greater asymmetry in the ring and little than in the index and middle fingers. The little finger was called asymmetrical in all cases. The loss of the ulnar wing tendon from the abductor digiti minimi was observed on occasions, but the transverse binding to the extensor tendons was always strong. The index finger was labelled symmetrical in twelve cases, the middle in eleven, and the ring in five. In one hand the index and middle fingers appeared to have a somewhat heavier transverse component on the radial than the ulnar side, and in another hand (of a different body) a similar condition existed in the index finger.

Measurement of the distance between the radial and ulnar wing tendons and the middle of the extensor tendons seemed an attractive proposition, but this was only reasonable in the case of middle and ring fingers. The index and little fingers, having two extensor tendons, could be measured only by taking a point midway between the two tendons and this was considered unreasonable. Even in the case of the middle and ring fingers, the radial side measurement was somewhat spurious, as the border of the wing tendon is made up essentially of the lumbrical. This was accepted, however, as the interosseous always made a major contribution to it in the fingers concerned. The measurement was taken at the line of the metacarpophalangeal joint dorsally. The measurements gave a comparison of Radial 1.47 to Ulnar 1.0.

In ten cases clamps were applied to the wing tendons which were then distracted. In eight cases the expansion gave way first in the radial triangle. In the other two, one tore out of its clamp and therefore had 0 to be discarded, and one, which showed reasonable symmetry in morphology, stretched considerably on $\vec{F}$ both sides before giving way on the ulnar side.

These tests were on formalin-fixed material, but samples were obtained from unfixed material of the middle and ring fingers of an amputated hand. Both these expansions gave way first to the radial sides, where they appeared considerably thinner morpho- o logically.

\section{CLINICAL OBSERVATIONS}

Cases of ulnar deviation of the fingers usually show signs of ulnar dislocation of the extensor tendons. In extreme cases the tendon may come to lie in the ulnar $\vec{i}$ gutter. At operation it is usually obvious that the site of maximal disruption of the extensor expansion has $\vec{N}$ occurred at the radial side of the extensor tendon, in 은 the area of thinness and weakness demonstrated anatomically. Where there is a large mass of synovium $>$ it may bulge through the tendon at this region, giving the impression of a herniation.

The formal analysis of the state of the extensor $\vec{v}$ expansion in rheumatoid disease is not usuallo $N$ possible clinically, other than as a rather genera impression. It is possible at operation, but it must be appreciated that most operations on the metacarpor phalangeal joints are carried out for either early synovectomy before joint disruption has occurred, or for reconstructive work.

Twenty cases have been operated on for surgery to the extensor expansion and metacarpophalangeal joint in which marked ulnar deviation of the fingers was present during the study period. Not all of the twenty cases had all the fingers of a single hand examined surgically, but this was done in seventeen cases. In all these cases there was some degree of shift of the extensor tendon to the ulnar side and in some it lay in the gutter between the joints. The expansion had stretched between the radial wing tendon and the extensor (Fig. 4). It was impossible to say that no ulnar-sided stretching had occurred; it had undoubtedly done so in some cases. But the radial disruption was by far the greater and the effect $N$ merely relative rather than absolute. In cases in which $N$ the extensor tendon lay in the ulnar gutter, the close $N$ attachment of the ulnar interosseous (or abductor $\omega$ digiti minimi) was always retained; virtually the whole lateral disruption in these cases occurred in the radial triangle.

Operations for synovectomy are of less value in analysis as these often take place before major disruption has occurred. At surgery in such cases, the extensor expansion showed a wide variation from an apparently normal, generally stretched normal, radial 


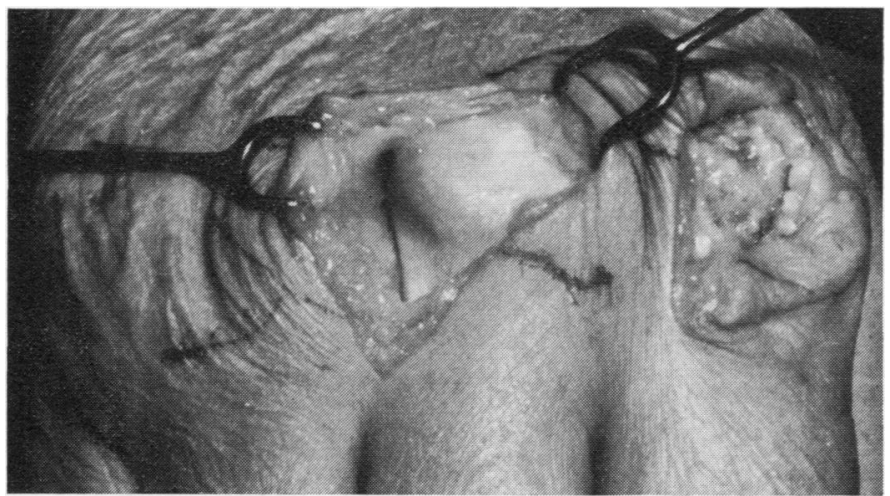

FIG. 4 An extensor expansion in a patient with ulnar deviation and volar subluxation. The extensor tendon can be seen lying to the ulnar side of the joint and the radial triangle of the expansion is grossly stretched.

stretch, and, on occasions, true herniation through the expansion in the radial triangle. No cases have been seen in which there was obvious ulnar-sided stretch or herniation.

Cases are sometimes seen in which the expansion, although perhaps stretched, remains more or less intact. There may be no evidence of giving way on either side (Fig. 5). In one such case there was severe

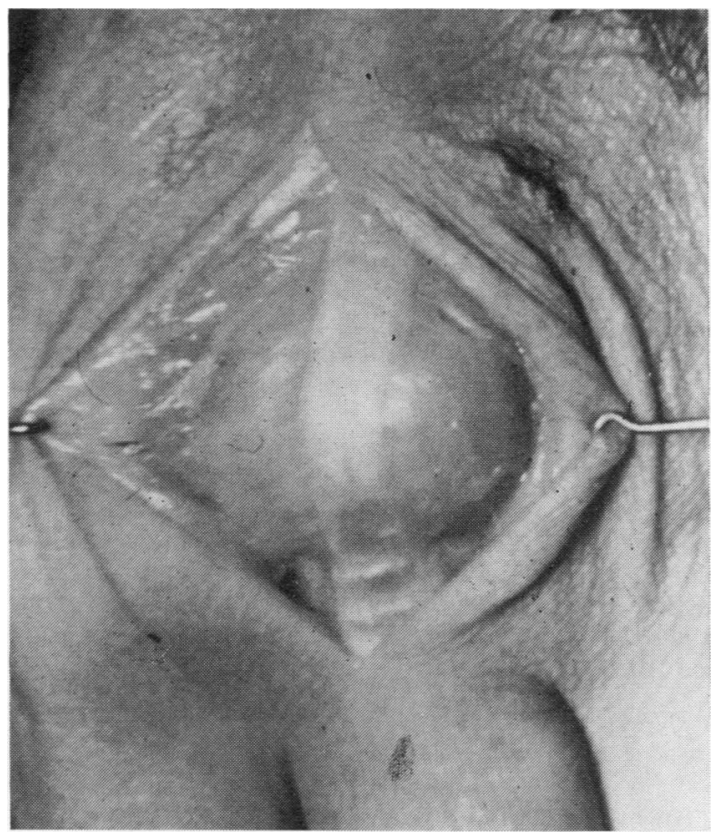

FIG. 5 A case in which there was gross destruction of the bones around the metacarpophalangeal joint and grossly stretched collateral ligaments, but no asymmetrical stretching of the expansion. There was no ulnar deviation and good digital control was retained.

underlying bone destruction, and after synovectomy the bone ends could be separated by over $1 \mathrm{~cm}$. with grossly stretched and thinned collateral ligaments. Nevertheless, these tightened up spontaneously after synovectomy, in the presence of an efficient expansion.

\section{Discussion}

The problems of assessing the causative factors of joint disruption in the rheumatoid hand are many. In our present state of knowledge the obvious facts are that there is increase of synovial bulk, which may be enormous, and that this lies within the joint capsule. The capsule and the bone are subjected to stress and, as a result of this and possibly as a direct result of the disease process, change occurs in these structures. In the metacarpophalangeal joint the ventral aspect consists of the volar plate with a synovial pocket at its proximal end. Laterally theo capsule is thickened by the collateral ligaments and the accessory collateral (or glenoid) ligaments; these are underlaid by synovial pockets which, on swelling, exert pressure on the collateral ligaments and the underlying bone. Brewerton (1965) has shown that the collateral ligaments become stretched with loss of lateral (and also volar) stability; the underlying bone may also become eroded. Dorsal to the collateral ligaments, the joint capsule is only a thin membrane and the extensor expansion acts as the effective dorsal capsule. This, being subject to the same stressing influences as the collateral ligaments, also stretches. As it is a rather more complicated structure than the collateral ligaments, the stretching varies proportionately with the intrinsic strength of the component parts.

Kuczynski (1971) has shown experimentally that during flexion the synovial pouches, if inflated, become very prominent dorsally and therefore directly load the extensor expansion. With increasing flexion of the finger, including interphalangeal joints, the expansion is carried distally and the thinner broader transverse components of the extensor expansion are brought into relation with the dorsal synovial pouches of the metacarpophalangeal joint over the metacarpal head. The extensor tendon forms a firm central mass and the interossei are variably attached to bone and collateral ligaments laterally as well as supplying contributions to the expansion. The model for disruption of the transverse fibres of the hood is set, if these fibres are, in fact, vulnerable to permanent stretching. 
Stretching of the expansion as of the collateral ligaments has been shown to occur in rheumatoid disease, and when this occurs it is most likely to be in the region of the thinner, broader radial triangle. Nevertheless, free stretching of the joint capsule may not occur in the disease. Castillo, El Sallab, and Scott (1965) have shown a reciprocal relationship between bone and soft tissue damage dependent upon the work output of the patient's hand. Likewise, in patients subjected to surgery on the metacarpophalangeal joints, there may or may not be evidence of gross stretching of the expansion in spite of serious bone damage. Conversely, in surgical patients in whom ulnar deviation was an important feature in the natural history of the disease, radial-sided disruption of the extensor expansion was a prominent feature in all the twenty cases examined in the series.

Patients in whom the extensor expansion shows no evidence of asymmetrical disruption may be those in whom exercise has maintained its intrinsic strength at the expense of the underlying bone. In others the expansion was originally more symmetrical, for both sides of the expansion and the collateral ligaments can become generally stretched, thus leading to volar subluxation without deviation.

No cases have been examined at operation in which radial deviation had occurred at the metacarpophalangeal joints, a condition occasionally found, particularly of the index and middle fingers. From the anatomical series described, it appears likely that the transverse fibres may sometimes be weaker to the ulnar side in.the index and middle fingers. One of the expansions subjected to distracting forces gave way first on the ulnar side after stretching somewhat on both sides. There might therefore be a relationship between radial deviation and ulnar-sided weakness of the expansion, as has been shown for the radial side in the more usual ulnar deviation. This possibility is however as yet unproven.

Essentially, the current concept is an extension of the original view of Brewerton, namely, dorsal as well as collateral collapse of the joint capsule. This leads to volar subluxation in the manner described by
Backhouse (1969) and to deviation to the mechanically dominant side (Backhouse, 1968; Hakstian and Tubiana, 1967). Thereafter, the pull of the extensor tendon is lost to the metacarpophalangeal joint, except as an ulnar deviator, to add to the possible deviating pull of the flexor tendons as postulated by Smith and others (1964), and Flatt (1968), and the more extensive zig-zag hand disruptions of Shapiro (1968), Pahle and Raunio (1969), and Stack and Vaughan-Jackson (1971).

\section{Summary}

The extensor expansion has been shown to exhibit considerable morphological asymmetry in the majority of anatomical specimens. The radial-sided transverse fibres of the hood overlying the metacarpophalangeal joint are commonly thinner and weaker, both visually and on test, than those of the ulnar side.

In rheumatoid disease the radial fibres are those more likely to be stretched and an essential correlation between ulnar drift and stretching of these fibres is shown. In the more severe cases there may even be a herniation of the dorsal synovial pouch through the weakened radial triangle of the extensor expansion. The extensor tendon may come to lie to the ulnar side of the joint through failure of the radial side of the expansion and possible maintained active pull of the ulnar-sided interosseous muscle (or the abductor digiti minimi in the case of the little finger).

I should like to thank my former students and colleagues at Charing Cross Hospital Medical School for their help in the anatomical studies, and particularly Prof. T. W. A. Glenister for the use of his dissection, which first attracted my attention to the asymmetry of the expansion. My clinical colleagues at St. Mary Abbots, the Royal London Homeopathic and West London Hospitals, particularly Drs. Kay and Coomes and Messrs. Churchill-Davidson and Kates, have been of great assistance, not only clinically, but also in discussion. The photography was by Miss P. M. Turnbull and Mr. R. Hutchings, and secretarial assistance was given by Miss V. Pearson and Miss M. Gill.

I acknowledge with thanks the support of the Arthritis and Rheumatism Council in this work.

\section{References}

BACKHouse, K. M. (1968) Ann. roy. Coll. Surg. Engl., 43, 154 (The mechanics of normal digital control in the hand and an analysis of the ulnar drift of rheumatoid arthritis)

(1969) Ann. rheum. Dis., 28, Suppl. p. 15 (Mechanical factors influencing normal and rheumatoid metacarpophalangeal joints)

Brewerton, D. A. (1965) 'The rheumatoid hand' in 'Progress in Clinical Rheumatology', ed. A. St. J. Dixon, p. 56. Churchill, London

Castillo, B. A., El Sallab, R. A., And Scott, J. T. (1965) Ann. Rheum. Dis., 24, 522 (Physical activity, cystic erosions, and osteoporosis in rheumatoid arthritis)

Flatt, A. E. (1968) 'The Care of the Rheumatoid Hand'. Mosby, St. Louis

Hakstian, R. W., AND Tubiana, R. (1967) J. Bone Jt Surg., 49A, 299 (Ulnar deviation of the fingers. The role of joint structure and function)

KUCZYNSKI, K. (1971) Hand, 3, 41 (The synovial structures of the normal and rheumatoid digital joints)

LANDSMeER, J. M. F. (1962) Ann. rheum. Dis., 21, 164 (Power grip and precision handling) 
NAPIER, J. R. (1956) J. Bone Jt Surg., 38B, 902 (The prehensile movements of the human hand)

PAHLE, J. A., AND RAUNIO, P. (1969) Ibid., 51B, 664 (The influence of wrist position on finger deviation in the rheumatoid hand)

SHAPIRO, J. S. (1968) Ibid., 50A, 634 (The etiology of ulnar drift: a new factor)

Smith, E. M., Juvinall, R. C., Bender, L. F., AND Pearson, J. R. (1964) Arthr. and Rheum. 7, 467 (Role of the finger flexors in rheumatoid deformities of the metacarpophalangeal joints)

Stack, H. G., AND Vaughan-Jackson, O. J. (1971) Hand, 3, 62 (The zig-zag deformity in the rheumatoid hand) 